\title{
Enhanced photoluminescence in gold nanoparticles doped ferroelectric liquid crystals
}

\author{
A. Kumar, ${ }^{1}$ J. Prakash, ${ }^{1,2}$ D. S. Mehta, ${ }^{2}$ A. M. Biradar, ${ }^{1, a)}$ and W. Haase ${ }^{3}$ \\ ${ }^{1}$ Liquid Crystal Group, National Physical Laboratory, Dr. K. S. Krishnan Road, New Delhi 110012, India \\ ${ }^{2}$ Instrument Design and Development Centre, Indian Institute of Technology Delhi, Hauz, Khas, \\ New Delhi 110016, India \\ ${ }^{3}$ Institute of Physical Chemistry, Technical University Darmstadt, Petersenstr. 20, 64287 Darmstadt, \\ Germany
}

(Received 28 April 2009; accepted 24 June 2009; published online 17 July 2009)

\begin{abstract}
We report the characterization and photoluminescence (PL) of newly synthesized deformed helix ferroelectric liquid crystal (DHFLC) material having short pitch and high spontaneous polarization. We observed ninefold enhancement in PL intensity in gold nanoparticles doped DHFLC material. This enhancement in the PL intensity has been attributed to the coupling of localized surface plasmon resonance from metal nanoparticles with DHFLC molecules, resulting in the increase in excitation and emission rate of the liquid crystal molecules in the localized electromagnetic field. These studies would provide a cutting edge tool in the realization of enhanced photoluminescent liquid crystal display devices. (c) 2009 American Institute of Physics. [DOI: 10.1063/1.3179577]
\end{abstract}

It has long been appreciated that liquid crystals (LCs) are the most promising materials for display as well as nondisplay (optical devices based on LC) applications in modern technology. ${ }^{1-3}$ The main drawbacks of the LC based display devices are their low brightness and energy efficiency due to the use of polarizers and absorbing color filters. The use of luminescent LC materials or photoluminescent sheets as active color filters has emerged as a fascinating approach toward the fabrication of luminescent LCDs. ${ }^{4-6}$ But it is quite difficult to make a luminescent LCD using pure LC materials as they emit less intense light in the visible region of the electromagnetic spectrum. ${ }^{7-9}$ Few attempts have been made to synthesize light emitting/electroluminescent LC materials with different aromatic cores, lateral substituents, and end groups. ${ }^{10,11}$ Enhancement in the intrinsic luminescence of the LC materials may certainly provide a better tool in the realization of emissive LCDs. Metal nanoparticles (MNPs) have attracted a great deal of attention due to their fascinating properties, tunability in electronic and electro-optical properties, and having the possibility of enhancing luminescence or fluoroscence. ${ }^{12-17}$ The gold nanoparticles (GNPs) and few dyes have been found to enhance fluorescence due to exhibition of localized surface plasmons (LSPs) and their interaction with fluorophores. ${ }^{18,19}$ The metal enhanced fluorescence phenomenon has also found many applications in biological sciences. ${ }^{20-22}$ The doping of MNPs in LCs has attracted a great deal of attention due to the enhanced electro-optical properties of the doped LC materials. But the effect of MNPs on the fluorescence of the LC materials has been reported rarely in literature. However, Kuo et l. $^{23}$ have observed the enhancement in luminescence intensity of nematic LC (5 CB) doped with silver NPs. The deformed helix ferroelectric LCs (DHFLCs), which are special kind ferroelectric LCs (FLCs), have been found very useful as they have much applications in display devices because of their low driving voltage, gray scale generation capability, easily achievable alignment, fast response,

\footnotetext{
a) Author to whom correspondence should be addressed. Electronic mail: abiradar@mail.nplindia.ernet.in.
}

etc. $^{24,25}$ Our group has demonstrated the nonvolatile memory effect based on GNPs doped DHFLC material (FLC 6304). ${ }^{26}$

In the present studies, we characterized newly synthesized DHFLC material, namely LAHS19, using different experimental techniques and discussed the enhancement of luminescence intensity in GNPs doped DHFLC material on the basis of interaction of DHFLC molecules with GNPs. It has also been found that the luminescence intensity of GNPs doped DHFLC material can be tuned by varying the concentrations of GNPs.

The GNPs were synthesized using citrate reduction method $^{27}$ and characterized by high-resolution transmission electron microscope (Technai G20-Stwin, USA). The typical size of GNPs prepared was around $15-20 \mathrm{~nm}$. For this study, homogeneously aligned cells (thicknesses of 3-5 $\mu \mathrm{m}$ ) of the DHFLC material (LAHS19) have been prepared. The various material parameters such as spontaneous polarization, rotational viscosity, and switching time of LAHS19 material have been measured using automatic LC tester (ALCT-P, Instec, USA). The transition temperature $\left(T_{c}\right)$ of the material has been measured by using Mettler Toledo differential scanning calorimetry (DSC) $822^{e}$ at a scanning rate of $2{ }^{\circ} \mathrm{C} / \mathrm{min}$. The luminescence excitation spectra were measured with Edinburgh Instruments F-900 spectrophotometer with spectral slit of width $1 \mathrm{~nm}$.

We have characterized the newly synthesized DHFLC material using various experimental techniques. Figure 1 shows the various material parameters such as spontaneous polarization $\left(P_{s}\right)$, rotational viscosity $(\eta)$, and response time of the material LAHS19 with applied voltage which have been observed at room temperature. The saturation value of $P_{s}$ [Fig. 1(a)] has been observed around $100 \mathrm{nC} / \mathrm{cm}^{2}$, while as the magnitude of rotational viscosity [Fig. 1(b)] has been observed at $700 \mathrm{mPa}$. The response time [Fig. 1(c)] was observed of the order of microseconds. The different phase assignments and corresponding transition temperatures were determined at atmospheric pressure by DSC. One can see various humps corresponding to the phase transitions from Fig. 2. The different phases of the material studied have been marked in the DSC thermogram itself. The DSC measure- 

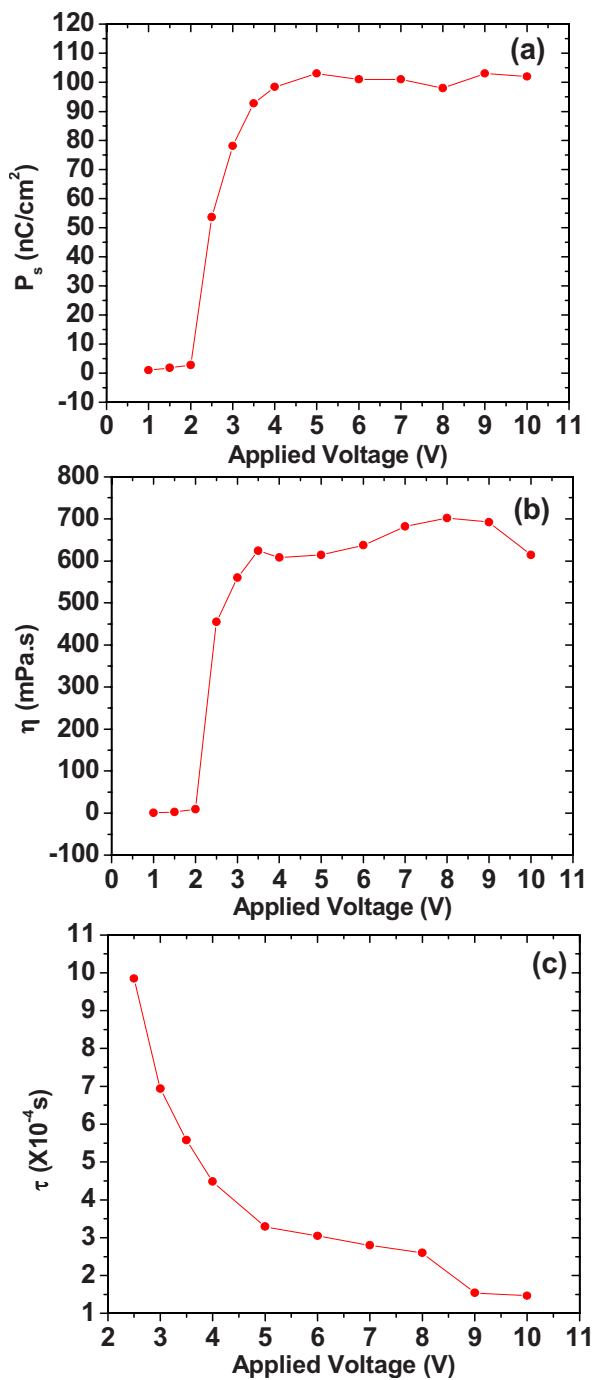

FIG. 1. (Color online) Behavior of (a) spontaneous polarization $\left(P_{s}\right)$, (b) rotational viscosity $(\eta)$, and (c) response time $(\tau)$ with applied voltage for DHFLC material LAHS19 at room temperature.

ments show that the $\mathrm{SmC}^{*}-\mathrm{SmA}^{*}$ phase transition temperature of the material studied is around $60{ }^{\circ} \mathrm{C}$. The material used, LAHS19, is a mixture of three phenylpyrimidine compounds, one biphenylpyrimidine, and chiral compounds. The molecular formulae and the concentration of these constituent compounds have been shown below.

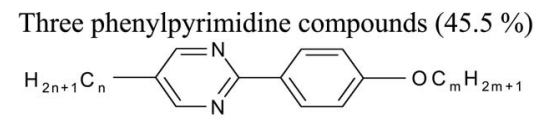

$m=6, n=8: 20.5 \%, m=n=8: 18 \%, m=8, n=9: 7 \%$

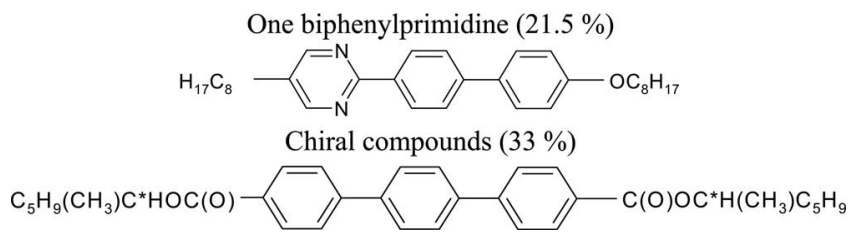

A large number of FLCs compositions have been synthesized earlier, which comprise at least two components and one of which is an optically active phenylpyrimidine compound. ${ }^{28}$ The derivatives of pyrimidine have been intensively investigated as electroluminescent materials as reported earlier. ${ }^{29,30}$ The blue photoluminescence (PL) emis-

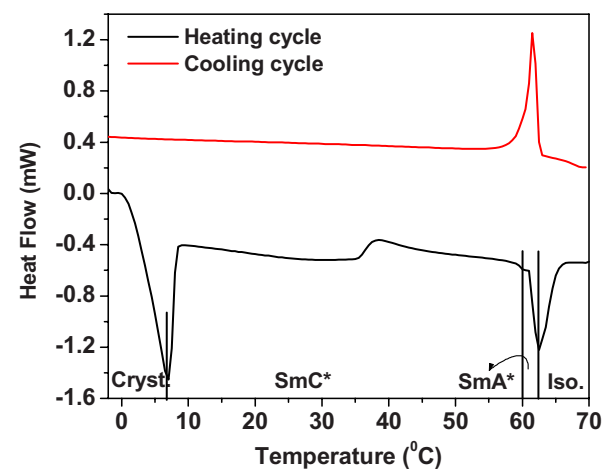

FIG. 2. (Color online) Differential scanning calorimetry graph of DHFLC material LAHS19.

sion due to highest occupied molecular orbital and lowest unoccupied molecular orbital energy levels of LC compounds containing heterocyclic pyrimidine has also been reported earlier. ${ }^{10}$

Figure 3 shows the emission spectra of LAHS19 material, which has been recorded at $1 \mathrm{~nm}$, slit width. The emission spectra in the spectral range of $350-650 \mathrm{~nm}$ from LAHS19 contain an intense violet peak at $411 \mathrm{~nm}$ with a comparatively weaker blue peak at $459 \mathrm{~nm}$. The absorption spectrum of DHFLC material has been shown in the inset of Fig. 3. One can clearly see that there is no any signature of PL from empty sample cell of indium tin oxide (ITO)-coated glass substrate. We have also studied the effect of the ordering of LC molecules on its PL spectrum. For getting unaligned sample, the sample cells were prepared without any surface treatment on the surfaces of ITO-coated glass substrates. It has been found that the molecular ordering does not affect the profile of PL but it is noticeable that the PL intensity of homogeneously aligned sample is greater than that of unaligned cell. It has also been investigated earlier that the luminescence intensity increases dramatically by highly scattering liquid crystalline phases. ${ }^{31}$ The MNPs are emerging candidates for enhancing the fluorescence intensity of the chromospheres lying in proximity to NPs surfaces due to generation of enhanced electromagnetic fields, when illuminated by light. Various concentrations of GNPs were doped in LAHS19 material to observe the effect of GNPs on the PL intensity of the GNPs doped LAHS19 material. The doping of GNPs solution in the LAHS19 material has been performed above the isotropic temperature of the material. The doping of GNPs has been carried out in steps with

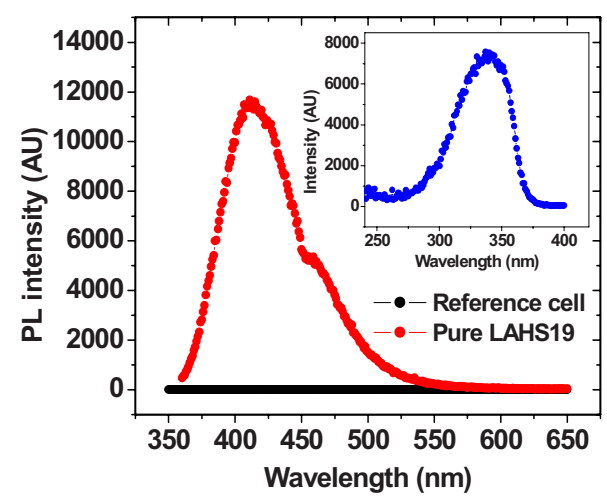

FIG. 3. (Color online) PL spectrum of DHFLC material LAHS19 and empty ITO cell, respectively, excited at $340 \mathrm{~nm}$, emission wavelength is $411 \mathrm{~nm}$. The absorption spectrum of DHFLC material LAHS19 has also been shown in the inset. 


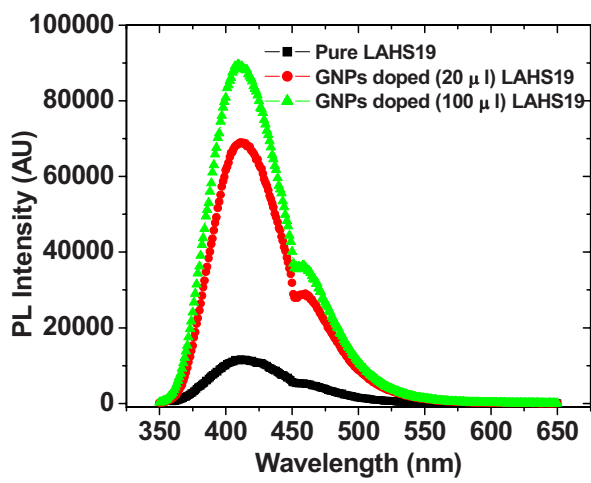

FIG. 4. (Color online) Comparison of PL intensity of pure DHFLC material LAHS19 and GNPs doped DHFLC material, excited at $340 \mathrm{~nm}$, emission wavelength is $411 \mathrm{~nm}$.

smaller amounts $(5 \mu \mathrm{l})$ of GNPs solution to prevent the aggregation of the GNPs within the material. The homogeneous distribution of the GNPs in the LAHS19 material ensures the possibility of the interaction between LSPs generated near the GNPs surfaces and the molecular fluorophores of the LAHS19 material. Figure 4 shows the PL spectra of LAHS19 doped with 20 and $100 \mu$ l of GNPs solution. One can clearly see sevenfold and ninefold enhancement in the PL intensity of LAHS19 with 20 and $100 \mu \mathrm{l}$ doping concentrations of GNPs solution, respectively. It is noticeable that the doping of GNPs enhances the PL intensity while they do not affect the PL profile of the LAHS19 material, as can be seen from Fig. 4. We have also observed the effect of doping of GNPs in conventional FLCs. The enhancement in the luminescence intensity is not remarkable in the case of conventional FLCs. The enhancement in the PL intensity of the GNPs doped LAHS19 material is due to the constructive interaction between enhanced electromagnetic field generated near the GNPs surfaces and molecular fluorophores (chiral terphenyl compound) present in the LAHS19 material. It has also been demonstrated earlier that the coupling of LSPs, generated near the NPs surfaces, with nearby fluorophore modifies the radiative decay rates and hence quantum yields of emission. ${ }^{32,33}$ In our case, the enhancement in the PL intensity of GNPs doped LAHS19 material is also due to increased radiative decay rates and quantum yields which happens because of the coupling of LSPs, generated near the NPs surfaces, with nearby fluorophore.

We have presented the results based on characterization of a DHFLC material and PL properties of GNPs doped DHFLC material. It has been observed that the material LAHS19 has short pitch, high spontaneous polarization, high viscosity, fast response, and larger intrinsic PL intensity. A ninefold enhancement in the PL intensity of the GNPs doped LAHS19 material has been observed. It has been demonstrated that generation of large electromagnetic fields near the GNPs surfaces enhances the PL intensity of GNPs doped LAHS19 material. The enhancement in the PL intensity of the GNPs doped LC material opens important ways in realization of photoluminescent LCDs. The LCDs based on the underlying phenomenon would have enhanced brightness, high contrast, large view angle, and less backlight scattering. The PL-LCD devices can be used in exceeding power efficiency of current LCDs, enhancing multiplexing in passive LCDs and for providing large screen direct view displays.
The authors sincerely thank Dr. Vikram Kumar, Director, NPL, New Delhi for continuous encouragement and interest in this work. The authors are also thankful to Dr. D. Harnath, LMD Section, NPL, New Delhi for taking PL measurements. We also sincerely thank to Mr. A. Lapanik, Technical University Darmstadt, Germany for providing DHFLC material. We acknowledge support for personal exchange via the Project No. IND 02/007 of BMBF, Germany and CSIR, India. The authors (A.K. and J.P.) are thankful to University Grants Commission, New Delhi and Council of Scientific and Industrial Research, New Delhi, respectively, for financial assistance.

${ }^{1}$ L. Marrucci, Liq. Cryst. Today 11, 6 (2002).

${ }^{2}$ H. Ren, Y. H. Fan, S. Gauza, and S. T. Wu, Appl. Phys. Lett. 84, 4789 (2004)

${ }^{3}$ D. W. Griffin, Opt. Lett. 26, 140 (2001).

${ }^{4}$ W. A. Crossland, I. D. Springle, R. D. King, P. A. Bayley, A. B. Davey, and B. Needham, Proc. SPIE 3955, 70 (2000).

${ }^{5}$ L. J. Yu and M. M. Labes, Appl. Phys. Lett. 31, 719 (1977).

${ }^{6}$ C. Weder, C. Sarwa, A. Montali, C. Bastiaansen, and P. Smith, Science 279, 835 (1998).

${ }^{7}$ Y. P. Piryatinskii and O. V. Yaroshchuk, Opt. Spectrosc. 89, 860 (2000).

${ }^{8}$ J. W. Y. Lam, Y. Dong, J. Luo, K. K. L. Cheuk, Z. Xie, and B. Z. Tang, Thin Solid Films 417, 143 (2002).

${ }^{9}$ Y. P. Piryatinskii, O. V. Yaroshchuk, L. A. Dolgov, T. V. Bidna, and D. Enke, Opt. Spectrosc. 97, 537 (2004).

${ }^{10}$ M. P. Aldred, A. J. Eastwood, S. P. Kitney, G. J. Richards, P. Vlachos, S M. Kelly, and M. O’Neill, Liq. Cryst. 32, 1251 (2005).

${ }^{11}$ M. P. Aldred, P. Vlachos, D. Dong, S. P. Kitney, W. C. Tsoi, M. O’Neill and S. M. Kelly, Liq. Cryst. 32, 951 (2005).

${ }^{12}$ S. Kaur, S. P. Singh, A. M. Biradar, A. Choudhary, and K. Sreenivas, Appl. Phys. Lett. 91, 023120 (2007).

${ }^{13}$ S. Eustis and M. A. El-Sayed, Chem. Soc. Rev. 35, 209 (2006).

${ }^{14}$ J. Lakowicz, C. Geddes, I. Gryczynski, J. Malicka, Z. Gryczynski, K. Aslan, J. Lukomska, E. Matveeva, J. Zhang, R. Badugu, and J. Huang, J. Fluoresc. 14, 425 (2004)

${ }^{15}$ S. Kuühn, U. Håkanson, L. Rogobete, and V. Sandoghdar, Phys. Rev. Lett. 97, 017402 (2006).

${ }^{16}$ T. Hartling, P. Reichenbach, and L. M. Eng, Opt. Express 15, 12806 (2007).

${ }^{17}$ J. Zhang and J. R. Lakowicz, J. Phys. Chem. B 109, 8701 (2005).

${ }^{18}$ S. Kalele, A. C. Deshpande, S. B. Singh, and S. K. Kulkarni, Bull. Mater. Sci. 31, 541 (2008).

${ }^{19}$ D. Ancukiewicz, NNIN REU Research Accomplishments (National Nanotechnology Infrastructure Network, Cornell, NY, 2008), p. 100.

${ }^{20}$ C. D. Geddes, H. Cao, I. Gryczynski, Z. Gryczynski, J. Fang, and J. R. Lakowicz, J. Phys. Chem. A 107, 3443 (2003).

${ }^{21}$ D. Rativa, A. S. L. Gomes, S. W. Hogiu, D. L. Farkas, and R. E. de Araujo, J. Fluoresc. 18, 1151 (2008).

${ }^{22}$ J. R. Lakowicz, B. Shen, Z. Gryczynski, S. D'Auria, and I. Gryczynski, Biochem. Biophys. Res. Commun. 286, 875 (2001)

${ }^{23}$ C. T. Kuo, S. Y. Huang, and C. C. Peng, Proceedings of the Abstract APS Meeting, 2007 (unpublished).

${ }^{24}$ L. A. Beresnev, V. G. Chigrinov, D. I. Dergachev, E. P. Poshidaev, J. Funfschilling, and M. Schadt, Liq. Cryst. 5, 1171 (1989).

${ }^{25}$ J. Funfschilling and M. Schadt, J. Appl. Phys. 66, 3877 (1989).

${ }^{26}$ J. Prakash, A. Choudhary, A. Kumar, D. S. Mehta, and A. M. Biradar, Appl. Phys. Lett. 93, 112904 (2008).

${ }^{27}$ J. Turkevich, P. C. Stevenson, and J. Hillier, Discuss. Faraday Soc. 11, 55 (1951).

${ }^{28}$ M. Ushioda, K. Ohno, and S. Saito, European Patent No. 0313336 (26 April 1989).

${ }^{29}$ B. Liu, X.-L. Hu, J. Liu, Y.-D. Zhao, and Z.-L. Huang, Tetrahedron Lett. 48, 5958 (2007).

${ }^{30}$ B.-L. Lee and T. Yamamoto, Polymer 43, 4531 (2002).

${ }^{31}$ R. V. Deun, D. Moors, B. D. Fre, and K. Binnemans, J. Mater. Chem. 13, 1520 (2003)

${ }^{32}$ C. D. Geddes and J. R. Lakowicz, J. Fluoresc. 12, 2 (2002).

${ }^{33}$ J. R. Lakowicz, Y. Shen, S. D’Auria, J. Malicka, J. Fang, Z. Gryczynski, and I. Gryczynski, Anal. Biochem. 301, 261 (2002). 